\title{
ON REGULAR MODULES
}

\section{HUANYIN CHEN and W. K. NICHOLSON ${ }^{凶}$}

\author{
(Received 25 November 2010)
}

\begin{abstract}
Several characterizations are given of (Zelmanowitz) regular modules among the torsionless modules, the locally projective modules, the nonsingular modules, and modules where certain submodules are pure. Along the way, a version of the unimodular row lemma for torsionless modules is given, and it is shown that a regular ring is left self-injective if and only if every nonsingular left module is regular.
\end{abstract}

2010 Mathematics subject classification: primary $16 \mathrm{E} 50$.

Keywords and phrases: regular modules, locally projective modules, torsionless modules, nonsingular modules, self-injective rings, pure submodules.

\section{Introduction}

Given any module ${ }_{R} M$, the dual of $M$ is denoted $M^{*}=\operatorname{hom}\left({ }_{R} M, R\right)$. Note that $M^{*}$ becomes a right $R$-module as follows: if $\lambda \in M^{*}$ and $a \in R$, define $\lambda a: M \rightarrow R$ by $x(\lambda a)=(x \lambda) a$ for all $x \in M$. In 1972, Zelmanowitz [5] called an element $x \in M$ regular if $x=(x \lambda) x$ for some $\lambda \in M^{*}$, and he called $M$ a regular module if every element is regular. The following lemma is implicit in [5], and will be used several times below. We include a proof for completeness.

LEMMA 1.1. Let ${ }_{R} M$ be a module and let $x \in M$. Then:

(1) $x$ is regular if and only if $R x$ is a projective direct summand of $M$;

(2) if $x$ is regular, say $(x \lambda) x=x, \lambda \in M^{*}$, and if $e=x \lambda$, then $e^{2}=e \in R$ and $R x \cong R e$ where $x \mapsto e$ under the isomorphism. Moreover:

$$
M=R x \oplus W \quad \text { where } W=\{w \in M \mid(w \lambda) x=0\} .
$$

PROOF. (1) Define $\pi: R \rightarrow R x$ by $r \pi=r x$ for all $r \in R$. If $R x$ is projective then $\pi$ splits, that is, $\varphi: R x \rightarrow R$ exists with $\varphi \pi=1_{R x}$. If, furthermore, $M=R x \oplus N$, define $\lambda: M \rightarrow R$ by $(r x+n) \lambda=(r x) \varphi$. Then $x=(x \varphi) \pi=(x \varphi) x=(x \lambda) x$, so $x$ is regular. The converse of (1) follows from (2).

The research of the first author was supported by the Natural Science Foundation of Zhejiang Province (Y6090404), and the research of the second author was supported by NSERC (Canada) Grant A8075.

(C) 2011 Australian Mathematical Publishing Association Inc. 0004-9727/2011 \$16.00 
(2) Given $(x \lambda) x=x, \lambda \in M^{*}$, write $e=x \lambda$. Then $e^{2}=e(x \lambda)=(e x) \lambda=x \lambda=e$. Moreover, $(R x) \lambda=R e$ so $\lambda: R x \rightarrow R e$ is epic, and this map is monic because $(r x) \lambda=0$ means that $r x=r[(x \lambda) x]=0$. Hence $R x \cong R e$ is projective and $x \lambda=e$. Finally, since $y-(y \lambda) x \in W$ for every $y \in M$, we have $M=R x+W$; this is direct because $r x \in W$ means $0=(r x) \lambda x=r x$.

In this note we give several new characterizations of regular modules in terms of torsionless, locally projective, and nonsingular modules, and we investigate the relationship between regularity and pure submodules. Throughout, modules will be left modules and homomorphisms will be written on the right, unless otherwise specified. If $N$ and $M$ are modules, the notation $N \subseteq{ }^{\oplus} M$ means that $N$ is a direct summand of $M$, and $N \subseteq$ ess $M$ means that $N$ is an essential submodule of $M$. The term 'regular ring' means a von Neumann regular ring.

\section{Torsionless modules}

A module ${ }_{R} M$ is called torsionless if it satisfies the following equivalent conditions:

(1) $\bigcap\left\{\operatorname{ker}(\lambda) \mid \lambda \in M^{*}\right\}=0$;

(2) ${ }_{R} M$ is isomorphic to a submodule of the direct product $R^{I}$;

(3) for some set $I, m M^{*}=0, m \in M$, implies that $m=0$.

Examples include projective modules and regular modules (if $x \neq 0$ and $x=(x \lambda) x$, $\lambda \in M^{*}$, then $x \lambda \neq 0$ ). Torsionless modules are studied in [4], and can be used to characterize classes of rings (for example, [3, Corollary 5.44]).

Torsionless right modules are defined in the same manner, and the dual $M_{R}^{*}$ of any module ${ }_{R} M$ is always torsionless. To see this, suppose that $M^{* *} \lambda=0$ where $\lambda \in M^{*}$; we must show that $\lambda=0$. But if $m \in M$, and if we define $\hat{m}: M_{R}^{*} \rightarrow R_{R}$ by $\hat{m}(\mu)=m \mu$ for all $\mu \in M^{*}$, then $\hat{m} \in M^{* *}$ so $m \lambda=\hat{m}(\lambda) \in M^{* *} \lambda=0$, as required.

Lemma 1.1 shows that $x \in M$ is regular if and only if $R x \subseteq \subseteq^{\oplus} M$ and $R x \cong R e$ for some $e^{2}=e \in R$; if $M$ is torsionless, we have the following 'dual' result.

THEOREM 2.1. The following conditions are equivalent for a module ${ }_{R} M$ :

(1) $M$ is regular;

(2) $M$ is torsionless and, for any $x \in M, x M^{*}=e R$ for some $e^{2}=e \in R$.

Proof. (1) $\Rightarrow(2)$. Let $x \in M$ and write $x=(x \lambda) x$ for some $\lambda \in M^{*}$. If $x \neq 0$ then $x \lambda \neq 0$, proving that $M$ is torsionless. Now write $e=x \lambda$. Then $e^{2}=e \in x M^{*}$, so $e R \subseteq x M^{*}$. Conversely, if $\gamma \in M^{*}$ then

$$
x \gamma=[(x \lambda) x] \gamma=(x \lambda)(x \gamma)=e(x \gamma) \in e R,
$$

and we obtain $x M^{*} \subseteq e R$, proving (2).

(2) $\Rightarrow$ (1). If $x \in M$, let $x M^{*}=e R, e^{2}=e \in R$. If $e=x \lambda, \lambda \in M^{*}$, it suffices to show that $e x=x$. But if $\mu \in M^{*}$ then $(x-e x) \mu=x \mu-e(x \mu)=0$ because $x \mu \in$ $x M^{*}=e R$. Hence $x-e x \in \bigcap\left\{\operatorname{ker}(\mu) \mid \mu \in M^{*}\right\}=0$ because $M$ is torsionless. 
Since projective modules are torsionless, the next result is clear by Theorem 2.1 and the fact that every principal, projective left module is isomorphic to $R e, e^{2}=e \in R$.

COROLLARY 2.2. The following are equivalent for a projective module ${ }_{R} P$ :

(1) $P$ is regular;

(2) for each $x \in P, R x$ is projective and $x P^{*}=e R$ for some $e^{2}=e \in R$;

(3) for each $x \in P, R x$ is a direct summand of $P$.

If $P$ is a projective module, conditions (2) and (3) are sometimes referred to as the unimodular row lemma. The proof of Theorem 2.1 shows it holds when $P$ is only required to be torsionless, and so gives the following corollary.

Corollary 2.3 (General unimodular row lemma). If ${ }_{R} M$ is torsionless, the following conditions are equivalent for $x \in M$ :

(1) $x$ is regular;

(2) $x M^{*}=e R$ for some $e^{2}=e \in R$;

(3) $R x \subseteq \subseteq^{\oplus} M$ and $R x \cong R e$ where $e^{2}=e \in R$ and $x \leftrightarrow e$ under the isomorphism.

\section{Locally projective modules}

A module $M$ is called locally projective if it satisfies the following equivalent conditions.

(1) Given the diagram where $\alpha$ is epic and $F$ is finitely generated,

$$
\text { there exists } \gamma: M \rightarrow A \text { such that }\left.\gamma \alpha\right|_{F}=\left.\beta\right|_{F} .
$$

(2) $x \in x\left(M^{*} M\right)$ for each $x \in M$, that is, $x=\left(x \lambda_{1}\right) y_{1}+\cdots+\left(x \lambda_{n}\right) y_{n}$,

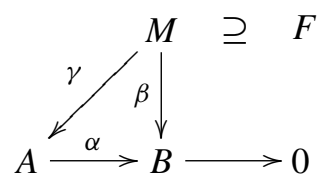

for some $\lambda_{1}, \ldots, \lambda_{n} \in M^{*}$ and $y_{1}, \ldots, y_{n} \in M$.

Here (1) is the original 1976 definition due to Zimmermann-Huisgen [6]; the characterization in (2) was proved in 1990 by Azumaya [1, Proposition 6]. Clearly every projective module is locally projective, and every finitely generated, locally projective module is projective. Condition (2) shows that every locally projective module is torsionless. Hence we have:

$\{$ regular modules $\} \subseteq\{$ locally projective modules $\} \subseteq\{$ torsionless modules $\}$.

These inclusions are strict: for the first, consider ${ }_{R} R$ where $R$ is not regular. For the second let $\mathbb{R}$ denote the field of real numbers, and consider the following subring of $\mathbb{R}^{\mathbb{R}}$ :

$$
R=\left\{\bar{x}=\left\langle x_{c}\right\rangle \in \mathbb{R}^{\mathbb{R}} \mid x_{c}=x_{c^{\prime}} \text { for all } c, c^{\prime} \in \mathbb{R} \backslash C_{\bar{x}}, \text { where } C_{\bar{x}} \subseteq \mathbb{R} \text { is countable }\right\} .
$$


Then $R$ is a regular ring and it follows from [6, Example 4.5] that $R^{\mathbb{R}}$ is not locally projective. Clearly $R^{\mathbb{R}}$ is torsionless.

If ${ }_{R} M$ is locally projective and $x \in M$, the right ideal $x M^{*}$ has a different description which motivated our discussion in the torsionless case. Write $x=\left(x \lambda_{1}\right) y_{1}+$ $\cdots+\left(x \lambda_{n}\right) y_{n}$ where $\lambda_{i} \in M^{*}$ and $y_{i} \in M$ for each $i$. We have $\sum_{i=1}^{n}\left(x \lambda_{i}\right) R \subseteq x M^{*}$ because $x M^{*}$ is a right ideal; and this is in fact equality because, for each $\beta \in M^{*}$,

Hence $^{1} x M^{*}=\sum_{i=1}^{n}\left(x \lambda_{i}\right) R$.

$$
x \beta=\sum_{i=1}^{n}\left(x \lambda_{i}\right)\left(y_{i} \beta\right) \in \sum_{i=1}^{n}\left(x \lambda_{i}\right) R .
$$

Since locally projective modules are all torsionless, Theorem 2.1 becomes the following.

THEOREM 3.1. The following are equivalent for a module ${ }_{R} M$ :

(1) $M$ is regular;

(2) $M$ is locally projective and, for any $x \in M, x M^{*}=e R$ for some idempotent $e \in R$.

Here is a direct proof of (2) $\Rightarrow(1)$ in the locally projective case. Given $x \in M$, let $x M^{*}=e R, e^{2}=e \in R$. As $M$ is locally projective, write $x=\sum_{i=1}^{n}\left(x \lambda_{i}\right) y_{i}, \lambda_{i} \in M^{*}$, $y_{i} \in M$. Then $x \lambda_{i} \in e R$ for each $i$, and it follows that $e x=x$. But $e \in x M^{*}$, say $e=x \beta$ where $\beta \in M^{*}$. Hence $(x \beta) x=e x=x$, proving (1).

As is well-known, every finitely generated right ideal of a regular ring is a principal ideal generated by an idempotent. The following proposition gives an analogue for modules.

PROPOSITION 3.2. The following are equivalent for a module ${ }_{R} M$ :

(1) $M$ is regular;

(2) $M$ is torsionless and, for any $x_{1}, \ldots, x_{n} \in M, \sum_{i=1}^{n} x_{i} M^{*}=e R$ for some $e^{2}=e \in R$;

(3) $M$ is locally projective and, for any $x_{1}, \ldots, x_{n} \in M, \sum_{i=1}^{n} x_{i} M^{*}=e R$ for some $e^{2}=e \in R$.

PRoOF. (2) $\Rightarrow(3)$ is clear, and (3) $\Rightarrow(1)$ by Theorem 3.1 .

$(1) \Rightarrow(2)$. We have seen that regular modules are torsionless. Given $x_{1}, \ldots, x_{n} \in$ $M$, we must show that $\sum_{i=1}^{n} x_{i} M^{*}=e R$ for some $e^{2}=e \in R$. Proceed by induction on $n$. If $n=1$ it follows by Theorem 2.1. If $n>1$ let $x_{n} M^{*}=f R, f^{2}=f \in R$. By induction let $\sum_{i=1}^{n-1}(1-f) x_{i} M^{*}=g R, g^{2}=g \in R$. Hence $f g=0$ therefore $e=f+g-g f$ is an idempotent, $f e=f=e f$, and $g e=g=e g$. Since $f x_{i} M^{*} \subseteq f R=x_{n} M^{*}$ for each $i$, we compute:

$$
e R=f R+g R=x_{n} M^{*}+(1-f) \sum_{i=1}^{n-1} x_{i} M^{*}=x_{n} M^{*}+\sum_{i=1}^{n-1} x_{i} M^{*} .
$$

This completes the induction and so proves (2).

\footnotetext{
${ }^{1}$ The notation $\left(x \lambda_{1}\right) R+\cdots+\left(x \lambda_{n}\right) R=o_{M}(x)$ is also used in the literature.
} 


\section{Nonsingular modules}

Recall that a ring $R$ is left self-injective provided that ${ }_{R} R$ is an injective $R$-module. LEMMA 4.1. If $R$ is a left self-injective ring, the following are equivalent for $a$ module ${ }_{R} M$ :

(1) $M$ is regular;

(2) every principal submodule of $M$ is regular;

(3) for any $x \in M, R x$ is projective and $x(R x)^{*}=e R$ for some $e^{2}=e \in R$.

PROOF. (1) $\Rightarrow(2)$. This is because submodules of any regular module are regular.

(2) $\Rightarrow$ (3). If $x \in M$ then $R x$ is regular by (2), so it is projective by Lemma 1.1. Now $x(R x)^{*}=e R$ where $e^{2}=e \in R$ by Theorem 2.1 .

(3) $\Rightarrow(1)$. Let $x \in M$. Since $R x$ is (locally) projective, there exist $\lambda_{1}, \ldots, \lambda_{n} \in$ $(R x)^{*}$ and $y_{1}, \ldots, y_{n} \in R x$ such that $x=\sum_{i=1}^{n}\left(x \lambda_{i}\right) y_{i}$. Moreover, $x(R x)^{*}=e R$ by (3) so $x \lambda_{i} \in e R$ for each $i$. It follows that $e x=x$. But $e \in x(R x)^{*}$, say $e=x \beta$ where $\beta: R x \rightarrow R$. Since ${ }_{R} R$ is injective, $\beta$ extends to $\lambda: M \rightarrow R$. Then $\lambda \in M^{*}$ and $(x \lambda) x=(x \beta) x=e x=x$, proving $(1)$.

If ${ }_{R} M$ is a module the singular submodule $Z(M)$ is defined by

$$
Z(M)=\left\{x \in M \mid l(x) \subseteq \subseteq_{R}^{\text {ess }} R\right\},
$$

where $I(x)=\{r \in R \mid r x=0\}$. We say that $M$ is nonsingular if $Z(M)=0$. Let $x \in \in_{R} M$ be regular, say $x=(x \lambda) x$ where $\lambda \in M^{*}$. If $x \in Z(M)$ then $x \lambda \in Z\left({ }_{R} R\right)$ because $I(x) \subseteq I(x \lambda)$. Since $x \lambda$ is an idempotent, this implies that $x \lambda=0$, whence $x=(x \lambda) x=0$. Hence regular modules are nonsingular, and the next theorem gives the converse for regular, left self-injective rings.

THEOREM 4.2. Let $R$ be a regular, left self-injective ring. The following conditions are equivalent for a module ${ }_{R} M$ :

(1) $M$ is regular;

(2) $M$ is nonsingular.

Proof. (1) $\Rightarrow(2)$. This always holds (see the discussion preceding this theorem).

$(2) \Rightarrow(1)$. Assume that ${ }_{R} M$ is nonsingular, and let $0 \neq x \in M$. Then $x \notin Z(M)$ so $I(x)$ is not essential in ${ }_{R} R$. Hence the set $\mathcal{S}=\{L \neq 0 \mid L$ is a left ideal, $L \cap I(x)=0\}$ is nonempty. By Zorn's lemma we may assume that $L$ is maximal in $\mathcal{S}$.

Claim. Lx $\subseteq$ ess $R x$.

Proof. Observe first that $L+1(x) \subseteq{ }^{\text {ess }}{ }_{R} R$. For if $[L+1(x)] \cap K=0, K \subseteq R$ a left ideal, it suffices to show that $(L+K) \cap 1(x)=0$ (then $L+K=L$ by the maximality of $L$, so $K=K \cap L=0)$. Suppose that $t \in(L+K) \cap 1(x)$, say $t=l+k$ where $l \in L, k \in K$. Then $l-t=-k=0$ by hypothesis, so $t=l \in L \cap l(x)=0$.

Now let $0 \neq a x \in R x$. We must find $c \in R$ such that $0 \neq c a x \in L x$. We may assume that $a x \notin L x$ (otherwise take $c=1$ ). Let $I=\{r \in R \mid r a \in L+I(x)\}$, a left 
ideal of $R$. Then $I a \subseteq L+I(x)$, and we claim that $I \subseteq{ }^{\text {ess }}{ }_{R} R$. For if $I \cap J=0$ where $J \subseteq R$ is a left ideal, it suffices to show that $[L+1(x)] \cap J a=0$ (then $J a=0$ by the preceding paragraph, so $J \subseteq I$ and hence $J=J \cap I=0)$. But if $j a \in L+I(x)$, $j \in J$, then $j \in J \cap I=0$, so $j a=0$.

Thus $I \subseteq{ }^{e s s}{ }_{R} R$. Since $M$ is nonsingular and $a x \neq 0$, this means that $I \nsubseteq I(a x)$, that is, $I a \nsubseteq I(x)$, say $c a x \neq 0, c \in I$. Since $c a \in L+I(x)$ it follows that $c a x \in L x$, proving the claim.

Now, since $L \cap I(x)=0$ the map $\varphi: L x \rightarrow R$ is well defined by $(a x) \varphi=a$ for all $a \in L$. Since $R$ is left self-injective, $\varphi$ extends to $\sigma: R x \rightarrow R$. But then $L x \cap \operatorname{ker}(\sigma)=0$, so $\sigma$ is monic by the claim.

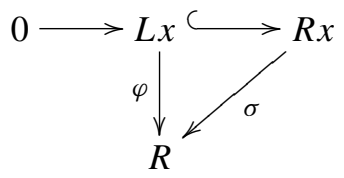

Since $R$ is regular, $R x \cong R(x \sigma)$ is projective, and so $R x$ has a dual basis $\left\{\lambda_{i} ; x_{i} \mid\right.$ $i=1,2, \ldots, n\}$. Then $x(R x)^{*}=\sum_{i}\left(x \lambda_{i}\right) R$ is a finitely generated right ideal of the regular ring $R$, and so $x(R x)^{*}=e R$ for some $e^{2}=e \in R$. It follows that $x$ is regular by Lemma 4.1, proving (1).

COROLLARY 4.3. The following are equivalent for a regular ring $R$ :

(1) $R$ is left self-injective;

(2) every nonsingular left $R$-module is regular;

(3) every finitely generated nonsingular left $R$-module is regular.

PROOF. (1) $\Rightarrow(2)$ is clear by Theorem 4.2, and (2) $\Rightarrow(3)$ is obvious.

(3) $\Rightarrow(1)$. Let ${ }_{R} M$ be a finitely generated, nonsingular left $R$-module. Then $M$ is regular by (3), and so is projective by [5, Corollary 1.7]. Hence (1) follows from [2, Theorem 9.2].

\section{Purity}

Recall that a submodule $N$ of a module ${ }_{R} M$ is called strongly pure in $M$ provided that for any $x \in N$, there exists an $R$-morphism $\alpha: M \rightarrow N$ such that $x \alpha=x$ (see $[1,6])$. Clearly every direct summand of $M$ is strongly pure in $M$.

LEMMA 5.1. The following are equivalent for a module ${ }_{R} M$ :

(1) $M$ is regular;

(2) every submodule of $M$ is strongly pure in $M$ and, for any $x \in M, R x \cong R e$, $e^{2}=e$, where $x \leftrightarrow e$ under the isomorphism.

Proof. (1) $\Rightarrow$ (2). Let $x \in N \subseteq M$. By (1) and Lemma 1.1, let $R x \oplus W=M$, and define $\alpha: M \rightarrow N$ by $(r x+w) \alpha=r x$ where $r \in R$ and $w \in W$. Then $x \alpha=x$ so $N$ is strongly pure in $M$. The rest of (2) follows by Corollary 2.3. 
(2) $\Rightarrow(1)$. Let $x \in M$ satisfy $R x \cong R e, e^{2}=e$, where $x \leftrightarrow e$ under the isomorphism. By Lemma 1.1 it suffices to show that $R x \subseteq{ }^{\oplus} M$. By (2), $R x$ is a strongly pure submodule of $M$, so there exists an $R$-morphism $\varphi: M \rightarrow R x$ such that $x \varphi=x$. If $\iota: R x \rightarrow M$ is the inclusion, then $\iota \varphi=1_{R x}$ so $M=R x \oplus \operatorname{ker}(\varphi)$, as required.

PROPOSITION 5.2. If ${ }_{R} M$ is a module, the following are equivalent:

(1) $M$ is regular;

(2) every submodule of $M$ is strongly pure in $M$ and, if $N \subseteq M$ is finitely generated, then $N \cong \bigoplus_{i=1}^{n} R e_{i}$ where $e_{i}^{2}=e_{i}$ and $R e_{i}$ is regular for each $i$.

PROOF. (1) $\Rightarrow$ (2). Every submodule of $M$ is strongly pure in $M$ by Lemma 5.1. Given $x_{1}, \ldots, x_{n}$ in $M$, it suffices to show that $\sum_{i=1}^{n} R x_{i} \cong \bigoplus_{i=1}^{n} R e_{i}$ where $e_{i}^{2}=e_{i} \in R$ and $R e_{i}$ is regular for each $i$. By Lemma 1.1, $R x_{1} \cong R e_{1}$ where $e_{1}^{2}=e_{1} \in R$, and $R x_{1}$ is regular being a submodule of a regular module. This proves (2) in the case where $n=1$. If $n>1$ write $N=\sum_{i=1}^{n} R x_{i}$ and $K=\sum_{i=2}^{n} R x_{i}$, so that $N=R x_{1}+K$. Now $N \subseteq{ }^{\oplus} M$ by [5, Corollary 1.3], so $N$ is regular by [5, Theorem 1.6]. By Lemma 1.1, it follows that $R x_{1} \subseteq{ }^{\oplus} N$, say $N=R x_{1} \oplus W$. But then

$$
W \cong N / R x_{1}=\left(R x_{1}+K\right) / R x_{1} \cong K /\left(K \cap R x_{1}\right)=\sum_{i=2}^{n} R\left(x_{i}+\left(K \cap R x_{1}\right)\right) .
$$

As $W$ is regular, it follows by induction that $W \cong \bigoplus_{i=2}^{n} R e_{i}$ where $e_{i}^{2}=e_{i} \in R$ and $R e_{i}$ is regular for each $i=2, \ldots, n$, so we are done using the case $n=1$.

(2) $\Rightarrow$ (1). If $x \in M$ then $R x$ is regular by (2) and [5, Theorem 2.8]. By Corollary 2.3 there exists $e^{2}=e \in R$ such that $R x \cong R e$ and $x \leftrightarrow e$ under the isomorphism. Since every submodule of $M$ is strongly pure in $M$, (1) follows from Lemma 5.1.

A submodule $N$ of a module ${ }_{R} M$ is said to be pure in $M$ if the inclusion $N \hookrightarrow M$ induces a canonical monomorphism $P \otimes_{R} N \rightarrow P \otimes_{R} M$ for any right module $P_{R}$. A well-known theorem of Cohn asserts that $N$ is pure in $M$ if and only if, whenever a finite system of equations $\sum_{j=1}^{n} r_{i j} x_{j}=n_{i}, n_{i} \in N, r_{i j} \in R$, has a solution $\left\{x_{i}\right\}$ in $M$, then it has a solution in $N$. As the names suggest, strongly pure submodules are pure. Indeed, suppose that the system $\sum_{j=1}^{n} r_{i j} x_{j}=n_{i} \in N$ has a solution $\left\{x_{j}\right\}$ in $M$. If for each $i, \alpha_{i}: M \rightarrow N$ satisfies $n_{i} \alpha_{i}=n_{i}$, then $\left\{x_{j} \alpha\right\}$ is a solution in $N$.

Proposition 5.3. A module ${ }_{R} M$ is regular if and only if it satisfies the following conditions:

(a) $M$ is locally projective;

(b) every principal submodule of $M$ is regular and pure in $M$.

PRoof. If $M$ is regular, (a) holds by Theorem 3.1, and (b) holds by Lemma 4.1.

Conversely, given (a) and (b), let $x \in M$. By (a) let $x=\sum_{j=1}^{n}\left(x \lambda_{j}\right) y_{j}, \lambda_{j} \in M^{*}$, $y_{j} \in M$. As $R x$ is a pure submodule of $M$, we can find $q_{1}, \ldots, q_{n} \in R x$ such that $x=\sum_{j=1}^{n}\left(x \lambda_{j}\right) q_{j}$. Hence the morphism $\alpha: M \rightarrow R x$ given by $\alpha=\sum_{i=1}^{n} \lambda_{i} q_{i}$ 
satisfies $x \alpha=x$, proving that $R x$ is strongly pure in $M$. As in the proof of (2) $\Rightarrow$ (1) in Lemma 5.1, this implies that $R x \subseteq \subseteq^{\oplus} M$. As $R x$ is regular by (b), it follows by Lemma 5.1 that $R x \cong R e$ where $e^{2}=e$ and $x \leftrightarrow e$. Hence $M$ is regular by Corollary 2.3.

\section{References}

[1] G. Azumaya, 'Some characterizations of regular modules', Publ. Mat. 34 (1990), 241-248.

[2] K. R. Goodearl, Von Neumann Regular Rings, 2nd edn (Krieger, Malabar, FL, 1991).

[3] W. K. Nicholson and M. F. Yousif, Quasi-Frobenius Rings, Tracts in Mathematics, 158 (Cambridge University Press, Cambridge, 2003).

[4] J. M. Zelmanowitz, 'Endomorphism rings of torsionless modules', J. Algebra 5 (1967), 325-341.

[5] J. M. Zelmanowitz, 'Regular modules', Trans. Amer. Math. Soc. 163 (1972), 341-355.

[6] B. Zimmermann-Huisgen, 'Pure submodules of direct products of free modules', Math. Ann. 224 (1976), 233-245.

HUANYIN CHEN, Department of Mathematics,

Hangzhou Normal University, Hangzhou 310036, PR China

e-mail: huanyinchen@yahoo.cn

W. K. NICHOLSON, Department of Mathematics, University of Calgary, Calgary, Canada T2N 1N4

e-mail: wknichol@ucalgary.ca 